\title{
V. PRELIMINARY STUDY ON ISOZYMES OF SHOREA JAVANICA
}

\author{
U. JUNIARTIand M. I. J. UMBOH
}

\author{
Tropical Forest Biology Program, BIOTROP, Bogor, Indonesia
}

\section{INTRODUCTION}

The detection of genetic variability in natural or man-made populations/ plantations is useful in both basic and applied biology. In addition to the various facets of studies on Shorea javanica already initiated by Torquebiau (1984) and alongside with his recommendations on focus for future research, a study on the genetic aspects of the species should be given important considerations. As the trees are tapped for resin, an important forest product, the genetic basis of the production as well as the range of variation in amount of resin production among the trees must be known. Coupled with this is a thorough investigation on the differences in pest resistance/susceptability among the trees and their genetic basis. While the assumption (Torquebiau 1984) that trees in natural forest areas are-rarely attacked by diseases because of mycorrhizal fungi is interesting, its confirmation is necessary. If this is true, problems would arise when plants are introduced into a new plantation site as experienced by the Forest Research Institute (Ardikoesuma 1954). Thus, we need to look for pest resistant plants i.e. those that can remain healthy even in the absence of mycorrhizae.

The above studies on possible genetic variation could give vital information for development of forest plantations of the species and for breeding and tree improvement strategies. By knowing the extent of genetic variation in natural population or in plantations one could be guided to maintain or increase the genetic base in these areas.

Biochemical characters such as isozyme banding patterns have been useful in several areas of plant biology, population genetics, evolution and breeding. Isozymes are detected by starch gel electrophoresis and when their genetic control is established, they could be genetic markers in analyzing variation in morphological or physiological characters.

The present study is an attempt to detect the isozymes in leaves, seeds and cotyledons of Shorea javanica by gel electrophoresis.

\section{MATERIALS AND METHODS}

Mature leaves from six mother trees and seeds from one tree were collected from Krui, Lampung (Sumatra) in September 1985. The leaves were kept inside a plastic ice cooling box immediately after harvest and stored in an incubator at $0^{\circ} \mathrm{C}$ until used for electrophoresis. Fifty mg of the leaf blade, seed or cotyledons of 
germinating seedlings were excised and ground in a mortar containing $20 \mathrm{mg}$ of quartz sand, $20 \mathrm{mg}$ of polyvinylpolyrrolidone (PVPP) and ca. $0.6 \mathrm{ml}$ of an extractant containing Triton- $\mathrm{x}-100$, TRis $(0.5 \mathrm{M})$, ascorbic acid $(0.5 \mathrm{M})$ adjusted to $\mathrm{pH} 7.0$ by acetic acid. Crude extract was absorbed into a $5 \mathrm{~mm} \times 9 \mathrm{~mm}$ Toyo No. 50 filter paper and the wicks were inserted into a $10 \%$ starch gel mediated with either $30 \mathrm{mM}$ borate buffer (pH 8.0) or $5 \mathrm{mM}$ histidine buffer ( $\mathrm{pH} \mathrm{6.6)}$ at the position of $10 \mathrm{~cm}$ from the anodal end of the gel. The gel was prepared following Smithies (1955) with a Toyo starch gel moulder. Electrophoresis was conducted with the use of a Toyo electrophoretic apparatus containing either $0.3 \mathrm{M}$ borate buffer $(\mathrm{pH} 8.0)$ or $0.6 \mathrm{M}$ sodium citrate $(\mathrm{pH}$ 6.2) at a constant voltage of $300 \mathrm{~V}$ for borate system and at $250 \mathrm{~V}$ for sodium citrate system. Running time was 3.5-4 hours in a Toyo Model IS-2200 incubator at $0^{\circ} \mathrm{C}$. The gel surface was covered with plastic wrap and chilled by ice throughout the run. Each gel was sliced horizontally with a sharp blade and gel slices were assayed for the following enzymes: Alcohol Dehydrogenase (after Schwartz and Endo 1966), Malic Acid Dehydrogenase (after Shaw and Prasad 1970), Peroxidase (after Endo 1978) and Superoxide dismutase (after Beauchamp and Fridovich 1971). The zymograms were recorded by drawing.

\section{RESULTS AND DISCUSSION}

\section{Alcohol Dehydrogenase (ADH)}

\section{A. Leaves}

Fig. 1 shows 3 anodally-moving bandmorphs. One tree showed bandmorph 1 consisting of 3 bands A1, A2, and A3. Another tree showed bandmorphs II consisting of 2 bands $\mathrm{A} 2$ and $\mathrm{A} 3$. Four trees showed a single band, A2.

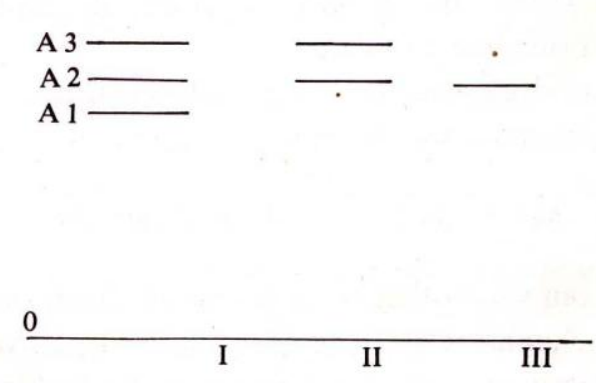

Fig. 1. ADH bandmorph in leaves. 


\section{B. Seeds}

Fig. 2 shows 5 anodally-moving bandmorphs. Out of 18 seeds, 5 showed bandmorph 1 consisting of 3 bands A1, A3 and A4; four showed bandmorph II consisting of bands A3 and A4; one showed bandmorph III consisting of band A4; four showed bandmorph IV consisting of bands A2, A4, A5 and A6 and four showed bandmorph $\mathrm{V}$ consisting of bands A1, A2, A4 and A5.

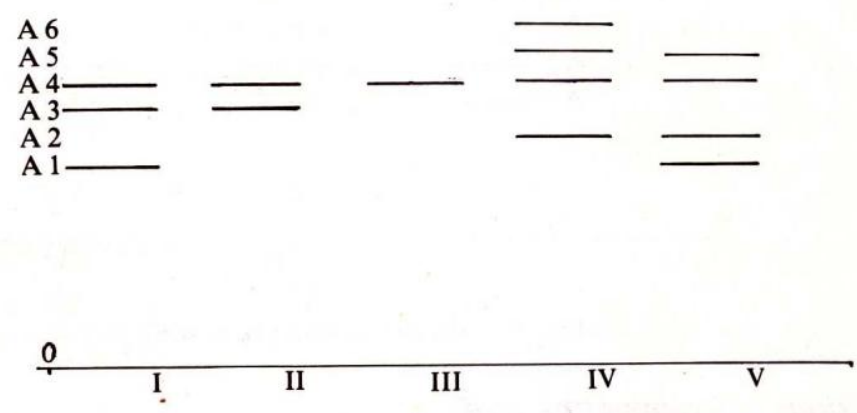

Fig. 2. ADH bandmorphs in seeds.

C. Cotyledons of germinating seeds

Only one ADH bandmorph was found, i.e. an anodally-moving Al band.

\section{Malate Dehydrogenase (MDH)}

\section{A. Leaves}

Fig. 3 shows 2 anodally-moving bandmorphs. Four trees showed bandmorph I with band A1, A3, A4, and A5 while two trees showed bandmorph II consisting of bands A1, $\mathrm{A} 2, \mathrm{~A} 3$ and $\mathrm{A} 4$.

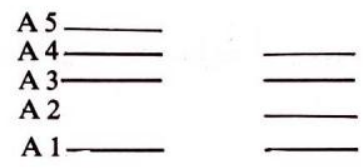

$\underline{0}$

I

II

Fig. 3. MDH bandmorphs in leaves. 


\section{B. Seeds}

Fig. 4 shows 3 anodally-moving bandmorphs. Twelve seeds showed bandmorph I consisting of bands A1, A2, A4 and A5; four seeds showed bandmorph II consisting of bands A1, A2, A3, A4 and A5; two showed bandmorph III consisting of bands A1, A2 and A5.

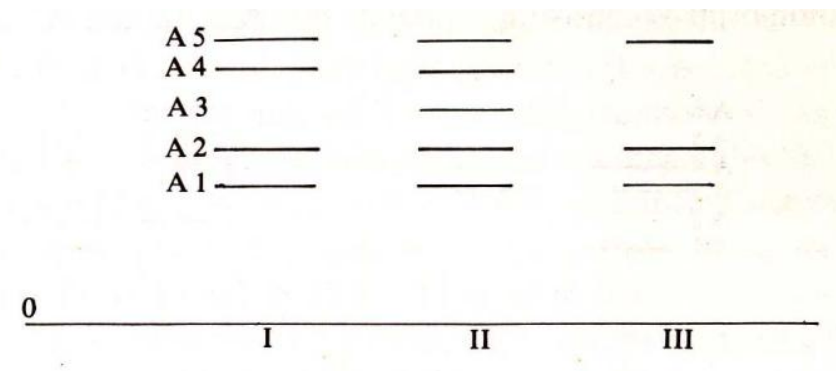

Fig. 4. MDH bandmorphs in seeds.

\section{Cotyledons of germinating seeds}

Variation in bandmorph was also found in cotyledons. Three MDH bandmorphs (Fig. 5) were found; all have 3 anodally-moving bands, but migration rates differ. Bandmorph I consists of bands A3, A5, A7; bandmorph II consists of bands A2, A4, A6 and bandmorph III consists of bands A1, A3 and A5.

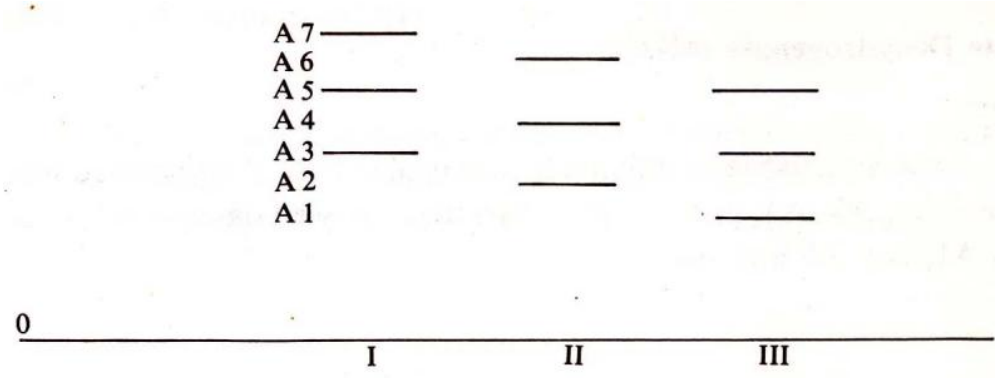

Fig. 5. MDH bandmorphs in cotyledons of germinating seeds.

\section{Peroxidase (POX)}

\section{A. Leaves}

Three bandmorphs were detected in the leaves (Fig. 6). These three have 4 similar cathodally-moving bands $\mathrm{Cl}, \mathrm{C} 2, \mathrm{C} 3$ and $\mathrm{C} 4$. Bandmorph $\mathrm{I}$ consists of 6 anodally-moving-bands A1, A2, A3, A4, A5 and A6. Bandmorph II consists of 5 anodally-moving bands A1, A2, A4, A5 and A6. Bandmorph III consists of 4 anodally-moving bands A1, A2, A4 and A6. 
V. Preliminary study on isozymes of Shorea javanica - Juniarti \& Umboh

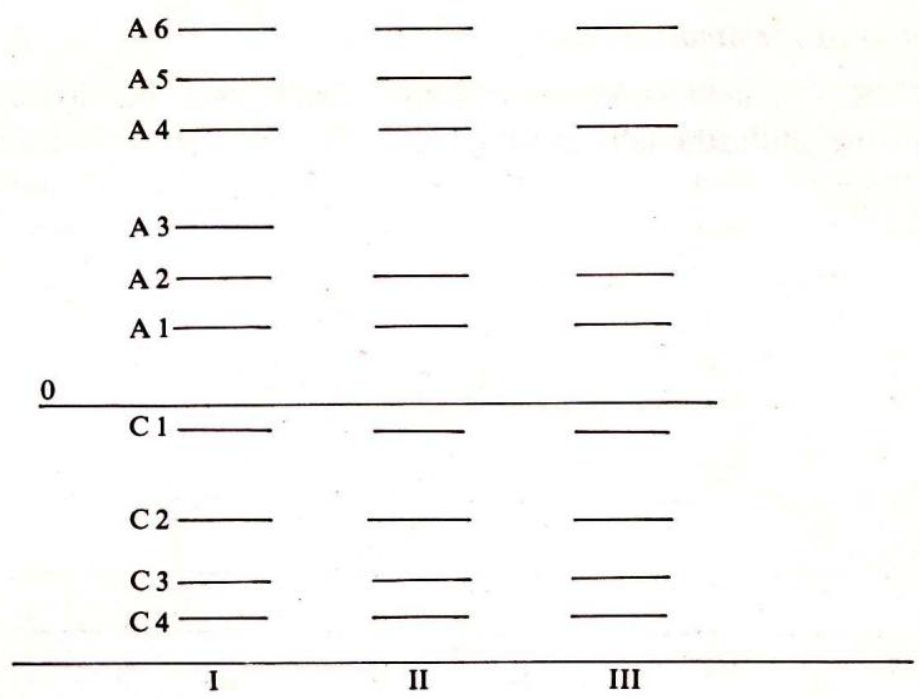

Fig. 6. POX bandmorphs in leaves.

\section{B. Seeds}

Eight different bandmorphs were detected in the seeds (Fig. 7), each one consisting of anodally and cathodally-moving bands. The range in total number of bands detected is 4-12.

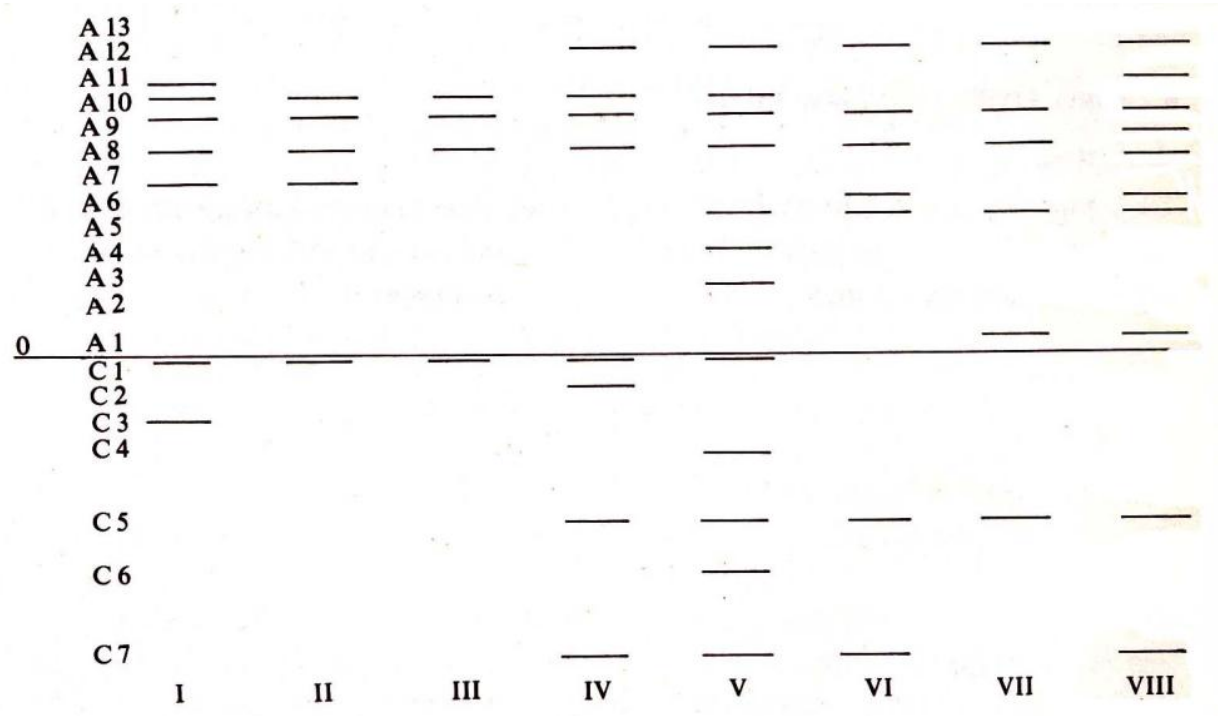

Fig. 7. POX bandmorphs in seeds. 


\section{Cotyledons of germinating seeds}

Eight POX bandmorphs were found; each one consisting both of anodally-moving and cathodally-moving bands (Fig. 8).

A 7

A 5

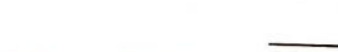




\section{B. Seeds}

Fig. 10 shows 4 bandmorphs. Eight seeds showed 4 anodally-migrating bands A1, A2, A3 and A4; two seeds showed 1 anodally-moving band A1; four seeds showed 4 anodally-moving bands A2, A3, A4 and A5 bands and 4 seeds showed one anodally-moving A2 band.

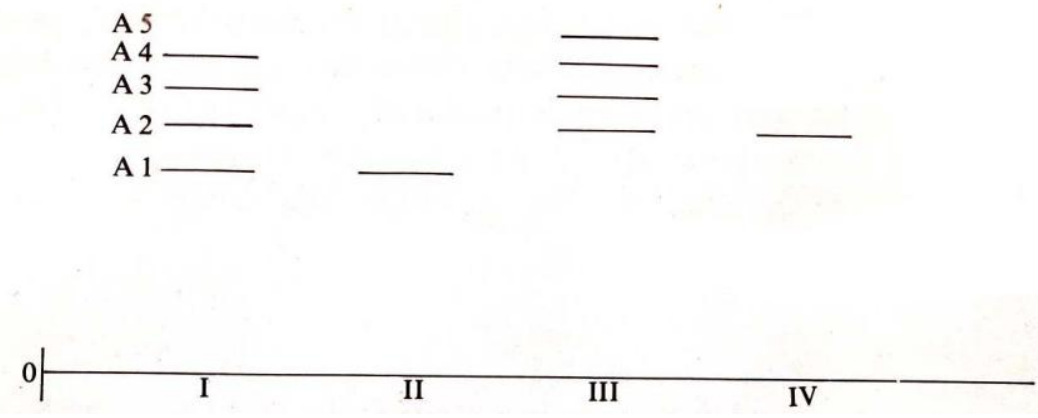

Fig. 10. SOD bandmorphs in seeds.

Following the method of starch gel electrophoresis, isozymes of 4 enzymes were detected as bands. The variation found in each of the enzymes assayed in leaves as well as among the seeds seems to be an indication of the diversity of this species in the plantations. However, considering the very limited sampling done from the plantation, the extent of this diversity could not be ascertained. Knowing the number of loci involved and the genetics of the observed differences will be prerequisites in understanding the genetic diversity. The mother tree of the seeds that were analyzed were inadvertently not recorded for its isozyme bandmorphs thus the variation in bandmorphs that was exhibited by the seeds could not be analyzed in relation with the bandmorphs of the mother tree. Otherwise, an insight into the breeding system of the tree could have been obtained.

Analysis of the isozymes in cotyledons of germinating seeds could possibly show differential gene activity at different developmental stages.

\section{SUGGESTIONS FOR FUTURE RESEARCH}

A sufficient number of trees must be sampled for isozyme detection from leaf extracts. To explain the genetic basis of the locus and its variation will require evidence from crosses between known bandmorphs. Since this is difficult to do with this large dipterocarp tree, a progeny test could suffice. This would involve collection of seeds per mother tree as basis of known bandmorph or genotype for the 6 enzymes and raising seedlings. Analysis of the enzyme pattern in the seedling leaves will have to be done. 
Sampling in a few plantation sites will be needed for the comparison of the genetic diversity among these sites. The analysis of the isozyme variation will provide data for quantifying the extent of the genetic variation in these sites.

A possible correlation could be searched between presence/absence of certain isozyme bands or whole genotypes and soil/habitat characteristics. It would be interesting to compare the frequency of certain isozyme alleles or whole genotypes among the sites. This may give a clue to the adaptive significance of some isozyme as found in some crop plants. When linkages are found between specific isozyme alleles and genes for economically important characters, e.g. growth, yield, pest resistance, susceptibility for mycorrhizal association etc., these isozyme markers could be used for selection of planting materials to establish plantations.

\section{NOTE}

The research described in this paper was originally carried out by Mrs. U. Juniarti and Dr. M.I.J. Umboh; the paper was written by Mrs. L.U. Gadrinab, whose assistance is gratefully acknowledged (editor).

\section{REFERENCES}

ARDIKOESOEMO, R.I. 1954. Tanaman Shorea javanica di Djawa. Rimba Indonesia 3-4 (1954), hal. 141-151.

BEAUCHAMP, C. and I. FRIDOVICH. 1971. Superoxide dismutase: Improved assays and assay applicable to acrylamide gels. Anal. Biochem. 44: 276.

ENDO, T., B.B. SHAHI and C. PAI. 1971. Genetic convergence of the specific acid phosphatase zymograms in Oryza saliva. Jpn. J. Genet. 46: 147.

1978. A new method for peroxidase isozyme stain. Ann. Rep. Nat. Inst. Genet. No. 28: 41.

GADRINAB, L. 1984. A biosystematic study on Section Pachycarpae in Dipterocarpaceae: Shorea macrophylla Ashton and Shorea stenoptera Burck. BIOTROP Internal Report.

GAN, Y. and F. ROBERTSON. 1981. Isozyme variation in some rain forest trees. BIOTROPICA 13 (1): 20-28.

IHARA, M. 1985. A report on forest genetic studies. BIOTROP Internal Report.

SCHWARTZ, D. and T. ENDO. 1966. The genetic control of alcohol dehydrogenase in maize-simple and compound loci. Genetic 53: 709.

SHAW, C.R. and E. PRASAD. 1970. Starch gel electrophoresis of enzymes. A compilation of recipes. Biochem. Genet. 4: 297.

SMITHIES, O. 1955. Zone electrophoresis in starch gels. Biochem. J. 61: 629.

TORQUEBIAU, E. 1984. Man-made dipterocarp forest in Sumatra. Agroforestry Systems, 2: 103-127. 